SF Osborne and $\mathrm{K}$ Jordan

Department of Ophthalmology

West Suffolk Hospital, Bury St Edmunds

IP33 2QZ, UK

Correspondence: SF Osborne

14 Heron Drive

Lenton, Nottingham

NG7 2DE, UK

E-mail: sarahfosborne@hotmail.com

Sir,

\section{Marcus Gunn syndrome}

Eye (2004) 18, 88-90. doi:10.1038/sj.eye.6700509

The Marcus Gunn syndrome, described by Gunn in 1883, is one of the more common congenital oculofacial synkineses, and accounts for approximately $5 \%$ of all congenital ptosis. ${ }^{1}$

Typically, the infant is noted soon after birth to have a ptotic lid, which elevates spontaneously when sucking or chewing. The lid elevates on contraction of the lateral (external) pterygoid muscle, which mediates opening, forward, and contralateral movement of the jaw. Less commonly, the lid elevates upon closure of the mouth, which is mediated by the medial (internal) pterygoid. A very rare variant, 'inverse' Marcus Gunn syndrome is characterized by the lid falling on opening the mouth, because of inhibiton of the levator muscle in association with lateral pterygoid contraction. Although bilateral cases of classical Marcus Gunn are well documented, there are, to our knowledge, only two previous reports of a patient with the classical syndrome on one side and an inverse Marcus Gunn synkinesis on the other. ${ }^{1,2}$ This report describes the case of a 4 year-old boy with what could be described as 'see-saw' Marcus Gunn syndrome.

\section{Case report}

A 4-year-old boy, presented with a right congenital ptosis. His mother was concerned about the cosmetic appearance of the lid as well as anomalous movements of the lids when eating. The child was otherwise well, had no history of other ocular problems, and there was no family history of similarly affected individuals.

Examination showed his visual acuities to be $6 / 9$ right 6/6 left. He had a 3-4 mm right ptosis, with typical Marcus Gunn syndrome on the right side, the lid elevating to the normal position with jaw opening. On the left side, the lid height was normal at rest, but became ptotic when the mouth was opened (Figure 1). There was no contraction of the orbicularis oculi. This resulted in 


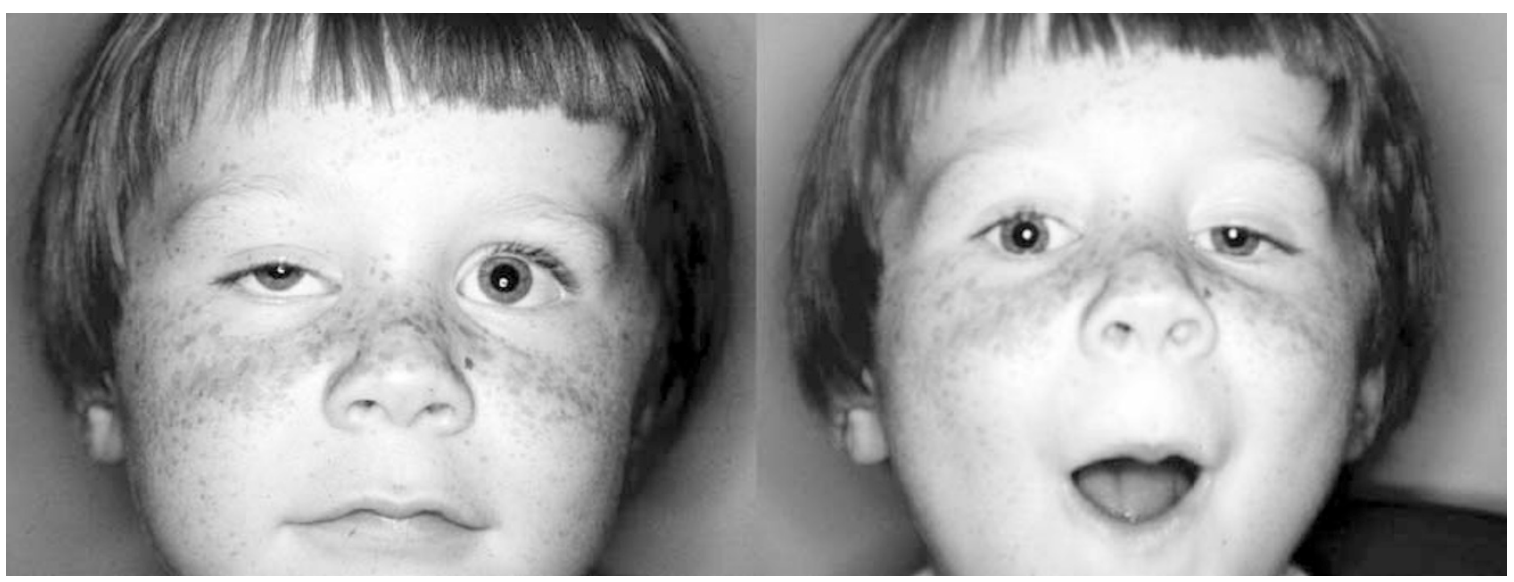

Figure 1 (left) Right congenital ptosis and left lid in normal position with the mouth closed. (right) Reversal of lid positions with the mouth open. 'See-saw' Marcus Gunn syndrome.

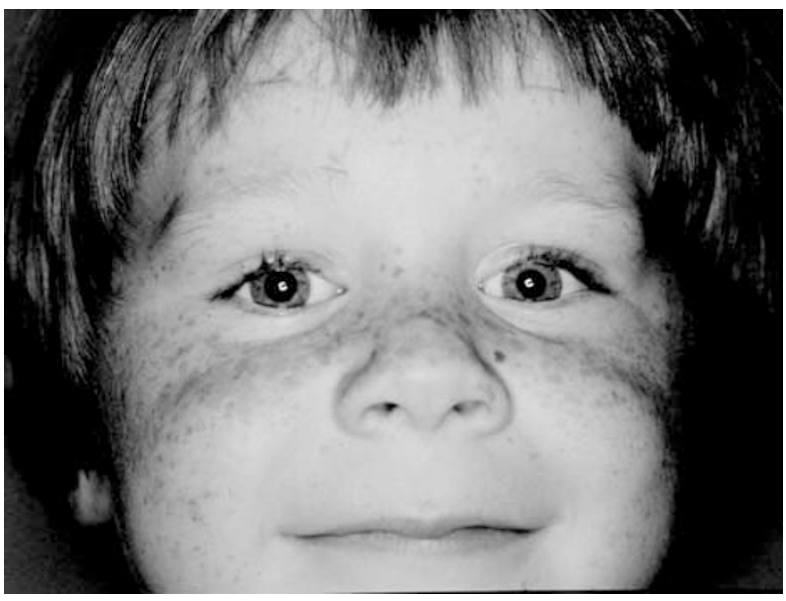

Figure 2 Postoperative appearance at 2 week.

the lids having a 'see-saw' motion with alternate opening and closing of the mouth. The voluntary levator function was right $3 \mathrm{~mm}$ left $10 \mathrm{~mm}$. Ocular movements were full and there were no other abnormal findings on ocular nor general examination.

The child underwent bilateral extirpation of the levator muscle and frontalis slings with autogenous fascia lata as described by Crawford, ${ }^{3}$ with a satisfactory functional and cosmetic outcome (Figure 2).

\section{Comment}

The Marcus Gunn syndrome is an interesting aetiological phenomenon. Electrophysiologic studies have documented cocontraction of the involved pterygoid and levator muscles in 'typical' cases, ${ }^{4}$ while one report documented inhibition of the levator muscle during ipsilateral lateral pterygoid contraction in a case of 'inverse' Marcus Gunn syndrome. ${ }^{5}$ Sano ${ }^{6}$ suggested, on the basis of electrophysiologic studies, that Marcus Gunn syndrome and other oculofacial synkineses represent release phenomena, as proposed by Pavesi et al. ${ }^{7}$ These phenomena are because of the reappearance of phylogenetically primitive synkinetic movements following injury to the involved cranial nerves in utero, with secondary degeneration of the cranial nerve nuclei. Cases may occur in isolation or in association with other anomalous ocular movements, such as double elevator palsy or Duane's syndrome, ${ }^{8}$ or with systemic anomalies such as cleft lip or palate. Bilateral cases have been reported in association with CHARGE syndrome. ${ }^{9}$ Such a heterogeneous group of patients suggests that the aetiologic mechanism is complex and that more than one factor is likely to be involved in the development of such synkineses.

This case adds further to the complexity, with the patient demonstrating the combination of both 'typical' and 'inverse' Marcus Gunn syndromes, that is, a positive synkinesis on one side and an apparent inhibitory synkinesis on the other, although the electrophysiologic basis of the anomalous movements was not investigated.

Inverse Marcus Gunn syndrome also needs to be distinguished from Marin Amat syndrome, where lid closure because of orbicularis oculi contraction is associated with mouth opening. ${ }^{1}$ Marin Amat syndrome generally follows seventh nerve palsy and is thought to be because of aberrant regeneration of the facial nerve. While some authors have used the terms 'inverse' Marcus Gunn syndrome and Marin Amat syndrome interchangeably, we believe the term inverse Marcus Gunn syndrome should be reserved for a congenital lesion, where lid closure is because of inhibition of the levator palpabrae superioris rather than orbicularis oculi contraction.

The treatment options in this case were more straightforward than in unilateral cases, as both lids 
required surgery to abolish the synkinesis and correct the subsequent ptosis. In this case, bilateral levator extirpation combined with frontalis suspension using autogenous fascia lata was successful in achieving the desired result.

\section{References}

1 Wartenberg R. 'Inverted Marcus Gunn phenomenon' (so called Marin Amat syndrome). Arch Neurol Psychiatr 1948; 60: 584-596.

2 Chattopadhyay A, Srinivas K, Sharatchandra B, Kannan N. The Marcus Gunn phenomenon: discussion and report of three cases. Quintessence Int 1995; 26: 563-566.

3 Crawford JS. Repair of ptosis using frontalis muscle and fascia lata: a 20-year review. Ophthalmic Surg 1977; 8(4): 31-40.

4 Pratt SG, Beyer CK, Johnson CC. The Marcus Gunn phenomenon: a review of 71 cases. Ophthalmology 1984; 91: 27-30.

5 Pavone P, Garozzo R, Trifiletti RR, Parano E. Marin-Amat syndrome : case report and review of literature. J Child Neurology 1999; 14: 266-268.

6 Sano K. Section of the portio minor of the trigeminal nerve for the treatment of the Marcus Gunn phenomenon. Mod Probl Paediatr 1976; 18: 258-262.

7 Pavesi G, Medici D, Macaluso GM, Ventura P, Allegri I, Genignani F. Unusual synkinetic movements between facial muscles and respiration in hemifacial spasm. Movement Disord 1994; 9: 451-454.

8 Creel D. Problems of ocular miswiring in albinism, Duane's syndome and Marcus Gunn phenomenon. Int Ophthalmol Clin 1984; 24: 165-176.

9 Weaver Jr RG, Seaton AD, Jewett T. Bilateral Marcus Gunn (jaw-winking) phenomenon occurring with CHARGE association. J Paediatr Ophthalmol Strab 1997; 34: 308-309.

\section{G Davis, C Chen and D Selva}

Oculoplastic and Orbital Unit

Department of Ophthalmology

Royal Adelaide Hospital

North Terrace, Adelaide SA 5000, Australia

Correspondence: D Selva

Tel: +61882225222

Fax: +6188222 5221

E-mail: raheyes@mail.rah.sa.gov.au

Sir,

Pseudo-Terson's syndrome: bilateral simultaneous rhegmatogenous retinal detachment Eye (2004) 18, 90-91. doi:10.1038/sj.eye.6700514

Vitreous haemorrhage secondary to subarachnoid haemorrhage was first described by Terson ${ }^{1}$ in 1900 . We report an unusual case of pseudo-Terson's syndrome presenting to Stoke Mandeville Hospital's Eye Casualty with bilateral spontaneous vitreous haemorrhages and occipital headache.

\section{Case report}

A 57-year-old man presented to the eye casualty with an acute onset of bilateral blurred vision, photopsia, associated with sudden onset of occipital headache, tinnitus, and fever. Nausea was the predominant symptom with no vomiting, neck stiffness, or neurological complaints. He was hypertensive, on atenolol and amitriptyline.

He was fully alert and oriented. Examination revealed a visual acuity of 6/9 in the right eye and 6/60 in the left eye. Retinal examination was obscured because of vitreous haemorrhage in both eyes. A provisional diagnosis of bilateral vitreous haemorrhage secondary to subarachnoid haemorrhage (Terson's syndrome) was made. A full neurological assessment excluded signs of meningeal irritation or neurological deficit. CT scan of the brain showed high signal in the superior sagittal sinus; however, it was reported as normal. Absence of an increased intracranial pressure and red blood cells or xanthocromia on lumbar puncture excluded subarachnoid haemorrhage. B-scan of orbits revealed dense vitreous haemorrhage in both eyes. Systematic survey of each of the eye's four quadrants did not show evidence of small retinal tears or rhegmatogenous retinal detachment in the peripheral fundi. Full blood count, biochemical profile, clotting profile, and electrocardiogram were all normal. Blood pressure was 141/95. MRI of the brain was reported normal.

Vitreous haemorrhage cleared rapidly by day 3 in both eyes. Ocular examination showed a horseshoe retinal tear with minimal subretinal fluid in the right superotemporal quadrant and a suspicious area in the left superotemporal quadrant. Right retinal detachment repair was performed together with cryotherapy to the left eye.

After 1 week later, examination revealed a second horseshoe retinal tear with local subretinal fluid in the right inferotemporal retinal quadrant and three horseshoe retinal tears and bullous retinal detachment in the left superotemporal quadrant. Neither macula was affected by retinal detachment. Visual acuity recovered to $6 / 12$ in the right eye and 6/6 in the left eye. Vitrectomy and fluid-gas exchange repair of the left retinal detachment and a second explant for the right eye were carried out.

\section{Comment}

Rhegmatogenous retinal detachment has a strong bilateral tendency. It results from chronic degenerative process, wherein both eyes are involved. The incidence of bilateral retinal detachment is $10 \%$ in normal population 